\title{
Correction to: The potential prognostic utility of salivary galectin-3 concentrations in heart failure
}

\author{
Xi Zhang $^{1}{ }^{\circledR} \cdot$ Nuwan Karunathilaka $^{1} \cdot$ Sameera Senanayake ${ }^{2} \cdot$ V. Nathan Subramaniam ${ }^{3} \cdot$ Wandy Chan $^{4}$. \\ Karam Kostner $^{5}$. John Fraser ${ }^{4}$ John J. Atherton ${ }^{6} \cdot$ Chamindie Punyadeera $^{1}(\mathbb{0}$
}

Published online: 10 December 2019

○) Springer-Verlag GmbH Germany, part of Springer Nature 2019

\section{Correction to: Clinical Research in Cardiology https://doi.org/10.1007/s00392-019-01557-0}

The original version of this article unfortunately contained a mistake.

The correct version of the Funding are given below:

\section{Funding}

Funding has been received from the National Heart Foundation of Australia Vanguard Grant.

1 Saliva and Liquid Biopsy Translational Research Team, School of Biomedical Sciences, Institute of Health and Biomedical Innovation, Queensland University of Technology, 60 Musk Avenue, GPO Box 2434, Brisbane, QLD 4001, Australia

2 Australian Centre For Health Services Innovation, Queensland University of Technology, Brisbane, QLD, Australia

3 School of Biomedical Sciences, Institute of Health and Biomedical Innovation, Queensland University of Technology, Brisbane, QLD, Australia

4 Critical Care Research Group, The Prince Charles Hospital, Brisbane, QLD, Australia

5 Department of Cardiology, Mater Adult Hospital, Brisbane, QLD, Australia

6 Cardiology Department, Royal Brisbane and Women's Hospital and University of Queensland School of Medicine, Brisbane, QLD, Australia 